\title{
High Resolution Simulation of Full Aircraft Control at Flight Reynolds Numbers
}

\author{
Scott A. Morton \\ US Air Force SEEK EAGLE Office (46 SK/SKI), Eglin \\ $A F B, F L$ \\ Scott.morton@eglin.af.mil
}

\author{
James R. Forsythe \\ Cobalt Solutions, LLC, Dayton, $\mathrm{OH}$ \\ \{forsythe,wurtzler\}@cobaltcfd.com
}

\author{
Kyle D. Squires \\ Arizona State University, Tempe, $A Z$ \\ squires@asu.edu
}

\begin{abstract}
This paper documents interim results of a three year project to develop a computational method for accurately determining static and dynamic stability and control characteristics of fighter and transport aircraft with various store configurations, as well as the aircraft response to pilot input. In this second year of the project computational data is gathered for a rigid F-16C with no control surface movement in forced motion that approximates flight test maneuvers. "Computational maneuvers" designed to efficiently gather three axes of motion data to build a comprehensive reduced order model are also developed. The data is then postprocessed to determine the resulting static and dynamic stability characteristics. The main benefits of this effort are: 1) early discovery of complex aerodynamic phenomena that are typically only present in dynamic flight maneuvers and therefore not discovered until flight test, and 2) rapid generation of an accurate aerodynamic database to support aircraft and weapon certification by reducing required flight test hours and complementing current stability and control testing.
\end{abstract}

\section{Introduction}

Practically every fighter program since 1960 has had costly nonlinear aerodynamic or fluid-structure interaction issues discovered in flight tests. The main reason for these "failures" is that the predictive methods used were not able to reveal the onset and nature of the problems early in the design phase. To keep the budget overshoot under control, fixes tend to be $a d$ hoc and are applied without a sound basis of fundamental understanding of the physics concerned. Unfortunately, in future aircraft designs, the problems will only become more complex as thrust vectoring, active aeroelastic structures, and other related technologies are implemented for stability and control augmentation. Unmanned combat vehicles will operate in flight regimes where highly unsteady, nonlinear, and separated flow characteristics dominate since there are no man-rating requirements ${ }^{[1]}$. In order to decrease the costs incurred by extensive flight-tests and the post-design phase modifications, it would be helpful to have a tool which enables aircraft designers to analyze and evaluate the nonlinear flight-dynamic behavior of the aircraft and/or associated armament, in the form of stability and control $(\mathrm{S} \& \mathrm{C})$ characteristics, early in the design phase.

The present paper provides an update on the firstyear effort to develop a high-fidelity simulation environment that will bring together aerodynamics, aeroelasticity and flight mechanics into a time accurate simulation tool. The benefits from such a tool to the areas of aircraft stability and control, flight simulation, and aircraft and weapon certification could potentially result in savings reaching into the billions of dollars ${ }^{[2]}$. The paper begins with a review of previous research in the field, followed by the objectives of this research. Next, the status of the tools being developed to support this effort is discussed. Finally, some preliminary results are presented. 


\section{Numerical Method}

This section presents the method of building an aircraft model suitable for determining the stability and control characteristics of fighter aircraft in the entire aircraft envelope. The first step in the method is to build a geometric representation of the complete aircraft of interest (including stores, control surfaces, inner loop control laws, aeroelastic effects, etc.). Next, simulations are performed of maneuvers designed to excite the relevant flow physics that will be encountered during actual missions in all three axes, roll, pitch, and yaw. These simulations are termed "computational maneuvers," since they may be unreasonable to fly due to actual aircraft or pilot limits. Next, a mathematical model is built of the aircraft response using system identification. Then, the model is tested by comparing computation fluid dynamics (CFD) simulations against model predictions of simulations expected to be encountered in flight. Finally, predictions of all flight test points are made using the model before flight tests are conducted to determine the expected behavior of the actual aircraft. Figure 1 depicts this process graphically. The following sub-sections describe the individual elements of the flow solver and system identification method necessary for the process.

\subsection{Flow Solver}

Computations are performed using the commercial flow solver Cobalt. Cobalt is a cell-centered, finite volume CFD code. It solves the unsteady, threedimensional, compressible Reynolds-Averaged NavierStokes (RANS) equations on hybrid unstructured grids. Its foundation is based on Godunov's first-order accurate, exact Riemann solver. Second-order spatial accuracy is obtained through a Least-Squares Reconstruction. A Newton sub-iteration method is used in the solution of the system of equations to improve time accuracy of the point-implicit method. Strang, et al. ${ }^{[3]}$ validated the numerical method on a number of problems, including the Spalart-Allmaras (SA) model, which forms the core for the Detached Eddy Simulation (DES) model available in Cobalt. Tomaro, et al. ${ }^{[4]}$ converted the code from explicit to implicit, enabling CFL numbers as high as $10^{6}$. Grismer, et al. ${ }^{[5]}$ parallelized the code, yielding linear speed-up on as many as 2,800 processors. The parallel METIS (PARMETIS) domain decomposition library of Karypis, et al. ${ }^{[6]}$ is also incorporated into Cobalt. New capabilities include rigid-body and six-degrees-offreedom (6DOF) motion, equilibrium air physics, Dynamic DES ${ }^{[7]}$ and overset grids in release Cobalt V4.0. A coupled aeroelastic simulation capability is also being developed.

\subsection{System Identification Analysis (SIDPAC)}

System identification (SID) is the process of constructing a mathematical model from input and output data for a system under testing, and characterizing the system uncertainties and measurement noises ${ }^{[8]}$. The mathematical model structure can take various forms depending upon the intended use. SID is usually applied to wind tunnel and flight test data to obtain accurate and comprehensive mathematical models of aircraft aerodynamics, for aircraft flight simulation, control system design and evaluation, and dynamic analysis. A very comprehensive review of SID applied to aircraft can be found in Morelli and Klein ${ }^{[9,10]}$ and Jategaonkar ${ }^{[11,12]}$.

Aircraft system identification can be used in cooperative approaches with CFD, to take advantage of the strength of both approaches or having one approach fill in the gaps where the other cannot be used effectively ${ }^{[9]}$. The wide range of SID tools that have been developed for aircraft system identification can easily be used to analyze CFD data computed for aircraft in prescribed motion. Here we follow the global nonlinear parameter modeling technique proposed by Morelli ${ }^{[13]}$ to describe the functional dependence between the motion and the computed aerodynamic response in terms of force and moment coefficients. The goal is to find a model which has adequate complexity to capture the nonlinearities while keeping the number of terms in the model low. The latter requirement improves the ability to identify model parameters, resulting in a more accurate model with good predictive capabilities. The modeling effort is global because the independent variables $(\alpha, \alpha \&$, $\beta$, etc.) are varied over a large range. Globally valid analytical models and their associated smooth gradients are useful for optimization, robust nonlinear control design and global nonlinear stability and control analysis.

\section{F-16 Results}

To date, a full-scale F-16 undergoing the following prescribed motions has been simulated: "computational maneuvers"-continuous $\alpha$ sweep, sinusoidal pitching, coning motion, oscillatory coning, configuration plunge pulse, plunge chirp, pitch chirp, Schroeder plunge, yaw chirp, composite pitch-yaw chirp, and flight test maneuvers - pitch doublet, and wind up turn. These motions represent both computational maneuvers and flight test maneuvers for stability and control testing and were defined using an interactive graphical user interface (GUI). Variations in Mach number from subsonic $(\mathrm{M}=0.3-0.6)$, transonic $(\mathrm{M}=0.85-0.95)$ to supersonic $(\mathrm{M}=1.2)$ were computed at a Reynolds number $(\mathrm{Re})$ of approximately 15 million. Many of the maneuvers have been covered in detail in previous papers (References 14- 
19), but the current paper will focus on various chirp signals and the flight test maneuvers not previously presented.

The grid used here for symmetric maneuvers is a half-span, full-scale model of the F-16 and non-symmetric maneuvers use the same grid mirrored about the symmetry plane. The model includes the forebody bump, diverter, and ventral fin. The engine duct is modeled and meshed up to the engine face. The wing-tip missile and corresponding attachment hardware are not modeled, however, nor is the nose boom. The three-dimensional (3D) hybrid grid was generated using the National Aeronautics and Space Administration, Langley grid generation packages GRIDToOL ${ }^{[20]}$ and VGRIDNS ${ }^{[21]}$, as well as the Cobalt L.L.C. grid management utility BLACKSMITH $^{[22]}$. The surface grid comprises 167,382 elements and off the surface there are eight prismatic layers. The height of the first prismatic layer corresponds to an average wall $y^{+}$value of less than four. In total there are 3.2 million cells with cells concentrated in the strake vortex. A full span grid with 6.4 million cells was created by mirroring cells across the symmetry plane for maneuvers that require a full span grid (e.g., coning, oscillatory coning). The boundary conditions are symmetry, adiabatic solid wall for the surface of the aircraft and the engine duct, and modified Riemann invariants for the far-field boundaries. A source boundary condition based on Riemann invariants is used to create an inflow condition at the engine exhaust. A sink boundary condition is used at the engine face to model the corrected engine mass flow.

The unsteady maneuvers were simulated using the DES, Spalart-Allmaras one-equation hybrid Reynoldsaveraged/large eddy simulation turbulence model with the streamline curvature and rotation correction (DES-SARC) to predict the effects of fine scale turbulence. Fully turbulent flow was assumed. The outer (physical) time step was set to $\Delta t=0.0004 \mathrm{~s}$, corresponding to a nondimensional time step (in terms of chord and freestream velocity) of $\Delta t^{*}=0.01$. The number of Newton subiterations was set to 5 . The unsteady numerical simulations were initialized by steady-state solutions computed with the Spalart-Allmaras RANS model with the streamline curvature and rotation correction (SARC).

The majority of the static validation computations were run on 64 to 128 processors on "Iceberg", an 800 processor IBM Power4 system operated by the Arctic Region Supercomputing Center (ARSC). All of the larger computations involving grid motion were run on 128-512 processors on either "Babbage", a 3,072 processor IBM Power5+ $(1.9 \mathrm{GHz})$ distributed-memory system operated by the Naval Oceanographic Office (NAVO) or "Jaws", a 5,120 processor Dell PowerEdge 1955 blade server cluster $(3.0 \mathrm{GHz}$, dual core) with Infiniband interconnect operated by the Maui High Performance Computing Center (MHPCC).

\subsection{Chirp Grid Motion Inputs}

Based on a cursory evaluation of a number of different motion types, it was determined that a simple chirp input applied to either a plunge or a rotational grid motion led to reduced order models with the best overall dynamic predictive capability. This is most likely due to the fact that the broad range of frequencies in the chirp signal excites the aerodynamic system over a large range of angle-of-attack, angle of sideslip, pitch rate, etc. Figure 2 depicts typical flowfield snapshots during a pitch chirp maneuver. The relationship used to create these chirp signals is the same as that used in the "chirp" function in MATLAB ${ }^{\circledR}$ and is given in Eq. (1).

$$
s(t) \quad \cos \left(2 \pi\left(\frac{\beta}{\lambda+1} t^{\lambda+1}+f_{1} t+\frac{\phi}{360}\right)\right) \text { where } \beta\left(\frac{f_{2}-f_{1}}{t_{2}^{\lambda}}\right)
$$

The parameters $f_{1}$ and $f_{2}$ denote the low and high limits of the chirp frequency bandwidth, respectively. The parameter $t_{2}$ is the time length of the chirp signal, and the parameter $\phi$ provides the ability to apply a phase shift to the signal as needed to help control whether or not the signal is biased relative to the starting amplitude. For a given signal length and bandwidth, the parameter, $\lambda$, controls the rate at which the signal traverses the requested frequency range. A value of $\lambda=1.0$ corresponds to a linear change in frequency whereas a value of $\lambda=2.0$ corresponds to a quadratic change in frequency, and so forth. Figure 3 shows the variation of frequency with time for a number of different values of this parameter.

Figure 4 shows chirp input signals for two different values of $\lambda$. The authors are currently investigating the effect of different values of the $\lambda$ parameter on the ability of models resulting from the various pitch chirp maneuvers to predict both static and dynamic validation data. Past experience has shown that a linear change in frequency in the chirp signal tends to result in poor model predictions of static data. Chirp signals with higher $\lambda$ values, which effectively dwell at the lower frequencies as seen in Figure 4, will hopefully improve these static predictions.

These various chirp signals have been used to drive both rotational and plunge motion of the CFD grid. While it is a fairly simple task to generate prescribed chirp motions about a single axis, it is more desirable to implement such motions in multiple axes so that the resulting models may be used to predict more complex motion (e.g., yaw-roll maneuvers). It is ultimately desired to be able to excite the aircraft aerodynamics based on motion in all three coordinate axes with a single CFD run. 
The resulting data could then be used to generate a loworder model for all six force and moment coefficients. Then, these models could be quickly differentiated to provide needed stability derivatives.

\subsection{Example Model Fit and Prediction}

As discussed above, it is desirable to use multipleaxis motions to generate more capable aerodynamic models versus just single-axis motions which would only be valid for a limited set of maneuvers and test cases. Tools have been developed to quickly generate multiple axis grid motions by combining multiple chirp signals to drive plunge or rotational motion. It is important for multiple input signals to be as "orthogonal" as possible in order to benefit the multivariate modeling process (i.e., make the resulting polynomial model terms more linearly independent). Therefore, the driver chirp signals for the multi-axis motion are made orthogonal via the $\lambda$ parameter discussed above. For example, in the case of a two-axis input motion, the $\lambda$ value for the first driver signal is specified, and the $\lambda$ value for the second signal is varied until the two signals are orthogonal (defined as a zero inner product).

Figure 5 shows an example of a combined pitch-yaw chirp motion designed to provide angle-of-attack motion between 0 and 30 degrees and sideslip motion between -15 and +15 degrees. The pitch chirp motion was generated with $\lambda=1.0$, and the yaw chirp motion was generated with $\lambda=1.47$ (computed to make the chirp signals orthogonal as discussed above). The left-pane of Figure 5 shows the required grid rotations about the three coordinate axes, and the right-pane shows the resulting angle-of-attack and sideslip excursions based on the prescribed flow conditions (0.6 Mach, 5,000 ft). Note that motion in all three coordinate directions is needed since the prescribed yaw motion in this case was about the vertical stability axis versus the vertical body axis. It is clear that this prescribed motion will force the aircraft through a large number of angle-of-attack/sideslip combinations (and likewise pitch, roll, and yaw rate combinations). This is a much more efficient way to "map" the flight envelope with aerodynamic force and moment coefficients versus running multiple static solutions.

Figure 6 shows the lift coefficient time history ("training data") plotted against angle of sideslip after implementing the grid motion in Figure 5 for the fullscale $\mathrm{F}-16 \mathrm{C}$ at $\mathrm{Mach}=0.6$ and 5,000 ft. Also shown in the figure is the resulting multivariate polynomial fit for lift coefficient using the SIDPAC software with angle-ofattack, angle of sideslip, roll rate, pitch rate, and yaw rate as model variables. The resulting model equation has the structure shown in Eq. (2), where the model terms are listed in order of most influential to least influential. This lift coefficient model results in a goodness of fit value of $99.86 \%$.

$$
\begin{aligned}
& C_{L}(\alpha, \beta, p, q, r) \quad C_{1}+C_{2} \alpha+C_{3} q+C_{4} p^{2}+C_{5} \alpha q^{2}+C_{6} \beta p q+ \\
& C_{7} \beta p+C_{8} \alpha^{2} q+C_{9} r+C_{10} \alpha \beta^{2}+C_{11} \alpha^{3}+C_{12} p r+ \\
& C_{13} \beta^{2} p+C_{14} \beta^{2} q+C_{15} p+C_{16} \beta^{2}
\end{aligned}
$$

As mentioned previously, the ultimate desire is to generate efficient yet accurate nonlinear aerodynamic models capable of predicting force and moment coefficients for both static and dynamic aircraft orientations. From these models, stability derivatives may be quickly calculated. Figure 7 shows predictions made with the model in Eq. (2) for both static lift curve data (left pane) as well as a pitch-axis oscillatory maneuver (linear chirp). Note that both of these validation data sets were generated from separate CFD runs (versus experimental data), so any errors or discrepancies may be assumed to be due to modeling errors or CFD convergence issues as opposed to vehicle configuration or flight condition differences. It is evident that both predictions match the validation data very well with the exception of the static high angle-of-attack data (left pane in Figure 7). This is most likely due to the small amount of low-frequency training data available at high angles of attack (due to the prescribed input motion). As mentioned above, chirp motions with different values of $\lambda$ are being investigated to try and improve these static predictions. Additionally, input motions traversing a larger angle-of-attack range (including post-stall) are currently being investigated.

\subsection{Flight Test-Derived Maneuvers}

The ultimate goal of the modeling process described above is to be able to accurately predict the stability (and eventually control) characteristics of the aircraft during realistic maneuvers. A number of flight test maneuvers such as wind-up turns, yaw-roll doublets, and steady heading sideslips have traditionally been used to evaluate the stability and flying qualities characteristics of an aircraft with a new weapons loading or configuration. Tools have been developed to derive the CFD grid motion needed to aerodynamically model the aircraft during such maneuvers. The left pane of Figure 8 shows the required grid motion to generate the same aerodynamic parameter histories (body axis rates, angle-of-attack, angle of sideslip) as an actual F-16C flight test maneuver accomplished by personnel at Eglin AFB, Florida. In this case, the flight test maneuver was a 2.5 -g wind-up turn. The right-hand pane of Figure 8 shows the measured (raw) angle-of-attack from the flight test overlaid with the angle-of-attack computed from the derived grid motion.

This maneuver plus a number of additional dynamic validation cases are currently being executed on "jaws" at 
the Maui High Performance Computing Center. The left pane of Figure 9 shows the required grid motion to emulate an actual pitch doublet flight test maneuver at Mach $=0.6$ and 5,000 ft (also performed at Eglin AFB, Florida). This grid motion implemented as part of a Cobalt flow solution to produce the validation data in the right pane of Figure 9. The polynomial lift coefficient model based on the pitch-yaw chirp maneuver discussed earlier was used to generate the predicted data in the right pane of Figure 9. The validation and prediction data yields a goodness of fit value of $99 \%$. Research into the ability of this and other models to predict various flight test-inspired maneuvers is ongoing.

\section{Conclusions and Outlook}

The status of a three-year project to develop a computational method for accurately determining static and dynamic stability and control characteristics of fighter and transport aircraft with various weapons configurations as well as the aircraft response to pilot input has been given. Now, just over half-way through the second year of the project, simulation results for the F-16C have been presented. This year marks a transition from computational simulations of simple motions in a single axis to complex maneuvers in all three axes and simulations of actual flight test maneuvers for verification. The results of the simulations and the proposed analysis process are showing extremely promising results and will result in significantly improved stability and control model building times over the traditional wind tunnel generated database approach, as well as, flexibility when encountering new configurations in the design phase. Comparisons of the results with flight test and wind tunnel data is on-going and will be presented in future papers.

In addition to the maneuver analysis documented in this paper, control surface implementation is occurring in a parallel effort to add control effectiveness characteristics to the analysis method. The first moving control surface simulations will occur in the last quarter of FY 07 and will be presented in the final year of the challenge. Additionally, in FY 08 the inner loop control laws will be added to the simulation to make the capability equivalent to actual flight test configurations. The method will be used to "shadow" an actual Air Force SEEK EAGLE certification of a store to show the validity of the method.

\section{FY 07 Related References and Awards}

There were four conference papers ${ }^{[15,17,18,19]}$, one Maui Applications Brief ${ }^{[16]}$, and two national awards ${ }^{[15,19]}$ associated with the Stability and Control project in FY 07:
Reference 15, Winner - ITEA National Symposium Best Poster Paper

Reference 19, Winner - AIAA Air Force Test and Evaluation Days Best Paper

\section{Acknowledgements}

This research was performed while a co-author held a National Research Council Research Associateship Award at the US Air Force Academy. In addition, this work was sponsored by the Department of Defense (DoD) HPC/Air Force SEEK EAGLE Office Institute for High Performance Computing Applications of Air Armament. The computational resources were generously provided by the DoD High Performance Computing Modernization Program, the Maui High Performance Computing Center, Aeronautical Systems Center, the Naval Oceanographic Office, and the Arctic Region Supercomputing Center. The authors gratefully acknowledge the expert advice of the following individuals: Kelly Cohen and Thomas Yechout at the US Air Force Academy; Eugene Morelli at National Aeronautics and Space Administration, Langley.

\section{References}

1. Withrow, M., "Dr. Raymond Gordnier Discusses the Research Direction of Advanced Computational Methods." Air Force Research Laboratory Horizons, April 2005.

2. Johnson, F.T., E.N. Tinoco, and N.J. Yu, "Thirty Years of Development and Application of CFD at Boeing Commercial Airplanes, Seattle." AIAA Paper 2003-3439, $16^{\text {th }}$ AIAA Computational Fluid Dynamics Conference, Orlando, FL, June 2003.

3. Strang, W.Z., R.F. Tomaro, and M.J. Grismer, "The defining methods of Cobalt 60 : a parallel, implicit, unstructured Euler/Navier-Stokes flow solver." AIAA Paper 1999-0786, 1999.

4. Tomaro, R.F., W.Z. Strang, and L.N. Sankar, "An implicit algorithm for solving time dependent flows on unstructured grids." AIAA Paper 1997-0333, 1997.

5. Grismer, M.J., W.Z. Strang, R.F. Tomaro, and F.C. Witzemman, "Cobalt: A Parallel, Implicit, Unstructured Euler/Navier-Stokes Solver." Adv. Eng. Software, Vol. 29, No. 3-6, pp. 365-373, 1998.

6. Karypis, G., K. Schloegel, and V. Kumar, "Parmetis: Parallel Graph Partitioning and Sparse Matrix Ordering Library, Version 3.1." Technical report, Dept. Computer Science, University of Minnesota, 2003.

7. Spalart, P.R., S. Deck, M.L. Shur, K.D. Squires, M.Kh. Strelets, and A. Travin, "A New Version of Detached-Eddy Simulation. Resistant to Ambiguous Grid Densities." Theoretical Computational Fluid Dynamics, Vol. 20, pp. 181195, 2006.

8. Ljung, L., System Identification. Theory for the User, Prentice Hall Information and System Sciences Series. 
9. Morelli, E.A. and V. Klein, "Application of System Identification to Aircraft at NASA Langley Research Center." Journal of Aircraft, Vol. 42, No. 1, pp. 12-25, January-February 2005.

10. Klein, V. and E.A. Morelli, "Aircraft System Identification Theory and Practice." AIAA Educational Series, 2006.

11.. Jategaonkar, R.V., "Flight Vehicle System Identification A Time Domain Methodology." Progress in Astronautics and Aeronautics, Vol. 216, F.K. Lu, editor, AIAA, 2006.

12. Jategaonkar, R.V., D. Fischenberg, and W. Gruenhagen, "Aerodynamic Modeling and System Identification from Flight Data - Recent Applications at DLR." Journal of Aircraft 2004, 0021-8669, vol.41, no.4, pp. 681-69, 2004.

13. Morelli, E.A., "Global Nonlinear Parametric Modeling with Application to F-16 Aerodynamics." ACC Paper WP04-2, Paper ID i-98010-2, American Control Conference, Philadelphia, Pennsylvania, June 1998.

14. Morton, S., S. Goertz, and D. McDaniel, "Computational Aircraft and Armament Stability and Control Techniques Applied to the F-16." ITEA Store Compatibility Symposium, Destin, FL, April 2006.

15. Morton, S.A., J. Dean, D.R. McDaniel, and S. Görtz, "Poster Paper on Computational Aircraft and Armament Stability and Control Techniques Applied to the F-16." International Test and Evaluation Association National Symposium, Orlando, FL, November 2006.

16. Morton, S.A., S. Görtz, D.R. McDaniel, "Computational Stability and Control Analysis of the F-16." Maui High Performance Computing Center Application Briefs, 2006.

17. Morton, S.A., McDaniel, D.R., and Cummings, R.M., "F16XL Unsteady Simulations for the CAWAPI Facet of RTO Task Group AVT- 113." AIAA Paper 2007-0493, Aerospace Sciences Meeting, Reno, NV, January 2007.

19. Görtz, S., D.R. McDaniel, and S.A. Morton, "Towards an Efficient Aircraft Stability and Control Analysis Capability using High-Fidelity CFD." AIAA Paper 2007-1053, Aerospace Sciences Meeting, Reno, NV, January 2007.

19. Dean, J., S.A. Morton, D.R. McDaniel, and S. Görtz., "Efficient High Resolution Modeling of Fighter Aircraft with Stores for Stability and Control Clearance." AIAA Paper 20071652, American Institute of Aeronautics and Astronautics Air Force Test and Evaluation Days Conference, Sandestin, FL, April 2007.

20. Samareh, S., "GridTool: A Surface Modeling and Grid Generation Tool, Proceedings of the Workshop on Surface Modeling, Grid Generation, and Related Issues in CFD Solutions." NASA CP-3291, May 9-11, 1995.

21. Pirzadeh, S., "Progress Toward A User-Oriented Unstructured Viscous Grid Generator." AIAA Paper 96-0031, 1996.

33. Blacksmith User's Manual, Cobalt Solutions, Version 3.0, 2005.

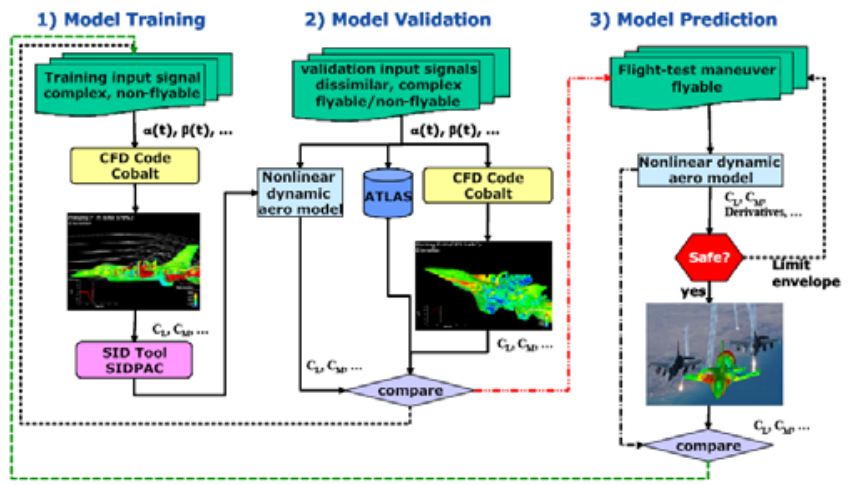

Figure 1. Stability and control model build process
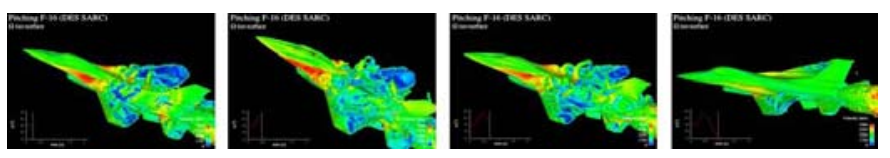

Figure 2. DES of F-16 in sinusoidal pitching motion of the initial stages of the pitch chirp; instantaneous vorticity isosurface colored by magnitude of velocity

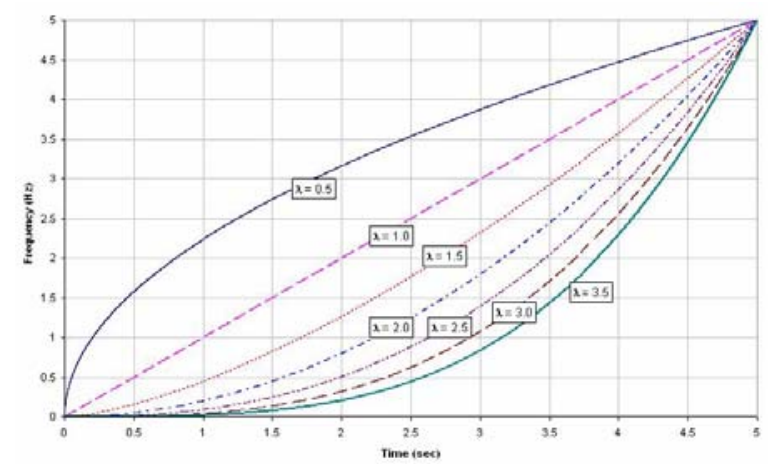

Figure 3. Frequency variation with time for various values of the chirp $\lambda$ parameter
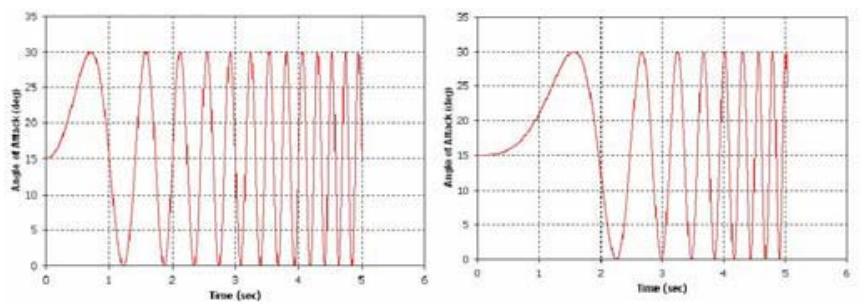

Figure 4. Angle-of-attack histories for single-axis chirp motions with $\lambda=1.0$ (left) and $\lambda=2.0$ (right) 

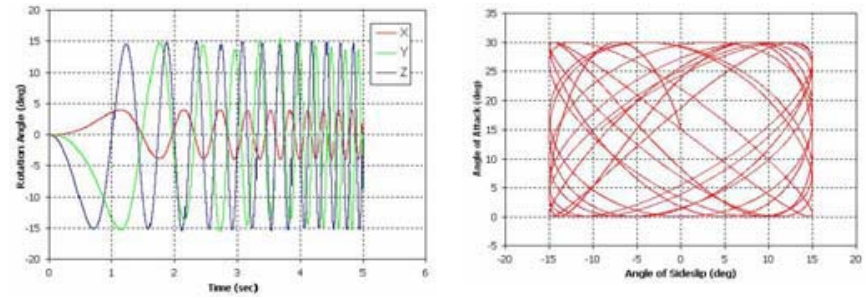

Figure 5. Grid motion (left) and resulting angle-ofattack/sideslip (right) for combined yaw-pitch chirp maneuver

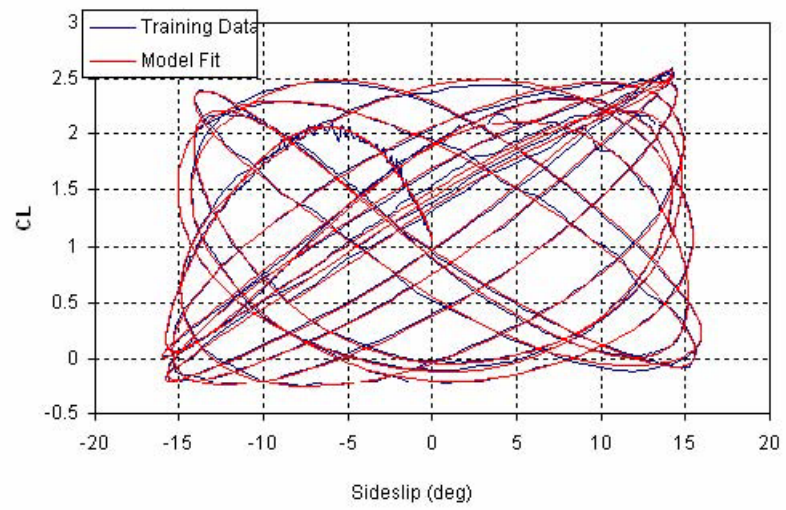

Figure 6: model fit for combined yaw-pitch chirp maneuver
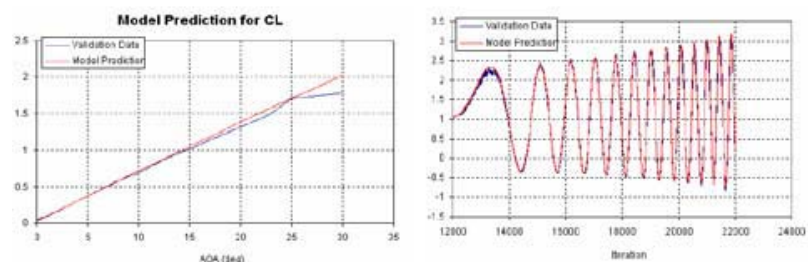

Figure 7. Model prediction of static (left) and dynamic (right) validation data
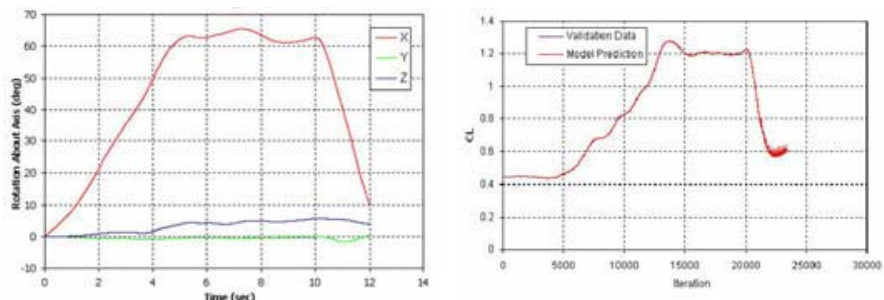

Figure 8. Grid motion (left) and angle-of-attack time history (right) for wind-up turn flight test maneuver
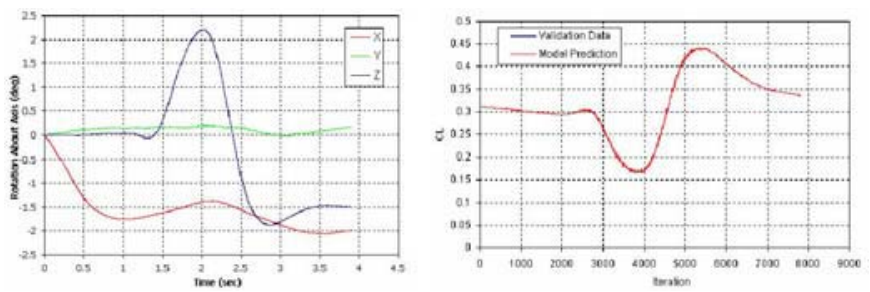

Figure 9. Grid motion (left) and model prediction (right) for pitch doublet maneuver 